\title{
Electronic structure of carbon nanotubes on graphene substrates
}

\author{
Benedetta Flebus ${ }^{1}$ and Allan H. MacDonald $\odot^{2}$ \\ ${ }^{1}$ Department of Physics and Astronomy, University of California, Los Angeles, Los Angeles, California 90095, USA \\ ${ }^{2}$ Department of Physics, The University of Texas at Austin, Austin, Texas 78712, USA
}

(Received 6 September 2019; revised manuscript received 25 March 2020; accepted 27 March 2020; published 15 May 2020)

\begin{abstract}
Allotropes of carbon, including one-dimensional carbon nanotubes and two-dimensional graphene sheets, continue to draw attention as promising platforms for probing the physics of electrons in lower dimensions. Recent research has shown that the electronic properties of graphene multilayers are exquisitely sensitive to the relative orientation between sheets and in the bilayer case exhibit strong electronic correlations when close to a magic twist angle. Here we investigate the electronic properties of a carbon nanotube deposited on a graphene sheet by deriving a low-energy theory that accounts for both rotations and rigid displacements of the nanotube with respect to the underlying graphene layer. We show that this heterostructure is described by a translationally invariant, a periodic, or a quasiperiodic Hamiltonian, depending on the orientation and the chirality of the nanotube. Furthermore, we find that, even for a vanishing twist angle, rigid displacements of a nanotube with respect to a graphene substrate can alter its electronic structure qualitatively. Our results identify a promising direction for strong correlation physics in low dimensions.
\end{abstract}

DOI: 10.1103/PhysRevResearch.2.022041

Introduction. Carbon nanotubes and graphene sheets are, respectively, one- and two-dimensional carbon allotropes. Both systems have been extensively studied for several decades because of their unique electrical, optical, and mechanical properties [1,2] and their wide range of potential applications spanning from electric circuits to solar cells and exciton-polariton lasers [3-6]. They have proven to be valuable platforms for investigating new physics in reduced dimensions, leading, for instance, to the first experimental observation of the integer quantum Hall effect at room temperature [7]. Graphene bilayers share many properties with their monolayer counterparts [8], but exhibit fascinating new phenomena as well [9]. Notably, Cao et al. and others have recently observed a correlated insulator state [10] and unconventional superconductivity [11] in graphene bilayers that have a relative orientation close to a magic [12] angle near $1.1^{\circ}$, sparking excitement in the physics community. Although they have not yet attracted wide attention, nanostructures formed between graphene and carbon nanotubes also hold promise since they might bring the sensitivity to orientations discovered in bilayers to one-dimensional physics.

In this Rapid Communication we establish a low-energy effective theory for hybridized nanotube-graphene heterostructures. We focus specifically on single-wall metallic nanotubes placed on a graphene layer, as illustrated in Fig. 1. We derive a Hamiltonian describing electron tunneling between a graphene layer and a nanotube deposited at a generic twist

Published by the American Physical Society under the terms of the Creative Commons Attribution 4.0 International license. Further distribution of this work must maintain attribution to the author(s) and the published article's title, journal citation, and DOI. angle. We find that, even for vanishing twist angle, rigid displacements of the nanotube with respect to the graphene sheet strongly alter the nanotube electronic properties, leading, e.g.,

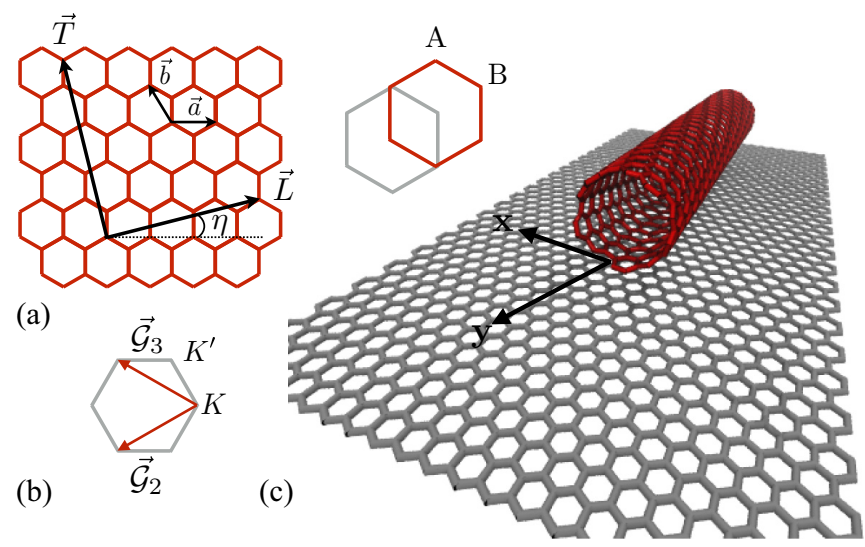

FIG. 1. Single-wall carbon nanotube on a graphene substrate. The nanostructure geometry can be characterized by starting from Bernal $A B$ stacking between a graphene sheet and an unrolled nanotube with general chirality and then displacing the nanotube or changing the orientation of its axis. In Bernal stacking, atoms located at the $B$ sites of the carbon nanotubes are above $A$ sites of the graphene layer. (a) Structure of an unrolled carbon nanotube, which is specified by a primitive translation vector $\vec{T}$ along the nanotube axis and a perpendicular chiral vector $\vec{L}$. Both vectors can be written as linear combinations of the primitive lattice vectors $\vec{a}$ and $\vec{b}$. (b) $\overrightarrow{\mathcal{G}}_{2}$ and $\overrightarrow{\mathcal{G}}_{3}$ are reciprocal lattice vectors that connect equivalent graphene Brillouin-zone corners. The momentum states near $K$ and its equivalent corners together host the Dirac point of valley $K$. Momenta near the other three Brillouin-zone corners host valley $K^{\prime}$, the time-reversed counterpart of valley $K$. (c) $A B$ stacking between graphene and the unrolled nanotube applies when the orientation of the nanotube axis matches its chirality angle $\eta$. 
to valley-dependent differences between the Dirac velocities of left and right movers, similar to the spin-dependent velocities induced in quantum wires by Rashba spin-orbit interactions [13]. For some magic displacements, nanotube group velocities in the vicinity of the Dirac points are strongly reduced, suggesting that strong interaction regimes and possibly [14] superconducting transitions might be made accessible by simple mechanical displacements.

Model. We consider a single-wall metallic nanotube deposited on top of a graphene layer, as illustrated in Fig. 1. The nanotube geometry is uniquely determined by the translational vector $\vec{T}=m_{a} \vec{a}+m_{b} \vec{b}$, oriented along the nanotube axis, and the chiral vector $\vec{L}=n_{a} \vec{a}+n_{b} \vec{b}$, with $L=|\vec{L}|$ the nanotube circumference. Here $\vec{a}$ and $\vec{b}$ are the primitive vectors of the graphene sheet's triangular lattice illustrated in Fig. 1 . The sets of integers $\left[n_{a, b}, m_{a, b}\right]$ are constrained by the orthogonality condition $\vec{L} \cdot \vec{T}=0$. Metallic nanotubes occur when the condition $n_{a}+n_{b}=3 N+1$ is satisfied, with $N$ an integer. For nanotubes with diameter larger than a few nanometers, we can safely neglect the effects of curvature of its wrapped graphene sheet $[2,15]$. In this case, the nanotube electronic states can be obtained by imposing the boundary condition $\psi(\vec{r})=\psi(\vec{r}+\vec{L})$ on the graphene wave functions, resulting in the momentum quantization condition $p_{x} \equiv$ $p_{x}(j)=2 \pi j / L$ [2], with $j$ an integer. We focus on the limit in which the interlayer tunneling strength is weaker than the energy separation between nanotube minibands, allowing us to truncate the low-energy Hilbert space to the $j=0$ subspace.

We consider an $A B$-stacked arrangement, as shown in Fig. 1, which can be achieved when the nanotube orientation on the graphene sheet matches the tube chirality angle $\eta$. The atomic positions in the graphene layer and the nanotube can be written, respectively, as $\vec{R}$ and $\vec{R}^{\prime}=M(\theta)(\vec{R}-\tau)+\vec{d}$, where the matrix $M(\theta)$ describes left-handed rotation of angle $\theta, \tau$ is a vector connecting the two atoms in the unit cell, and $\vec{d}$ is a translation vector. In our coordinate system, the nanotube axis is oriented along the $y$ direction. With these assumptions, the Dirac Hamiltonians of the carbon nanotube, $h\left(p_{y}\right)$, and the graphene layer, $h_{g}(\vec{k}, \eta, \theta)$, can be written as

$$
\begin{aligned}
h\left(p_{y}\right) & =-v\left[\begin{array}{cc}
0 & i p_{y} \\
-i p_{y} & 0
\end{array}\right], \\
h_{g}(\vec{k}, \eta, \theta) & =-v\left[\begin{array}{cc}
0 & k^{+} e^{-i(\eta-\theta)} \\
k^{-} e^{i(\eta-\theta)} & 0
\end{array}\right],
\end{aligned}
$$

where $\vec{k}$ (with $k^{ \pm}=k_{x} \pm i k_{y}$ ) and $p_{y}$ are momenta measured with respect to the respective Dirac points and $v$ is the Dirac velocity.

Here we assume that the overlap between the $\pi$ orbitals of the two subsystems can be represented by a function $t(\vec{r})$ that is smooth on the scale of the lattice spacing [12]. For the explicit calculation, we adopt a two-center approximation for the interlayer hopping amplitude [12] and account for the finite transverse size of the nanotube by assuming that the hopping amplitude varies with position in the $\vec{L}$ direction. ${ }^{1}$ For definiteness, we take $t(x) \propto e^{-x^{2} / 2 R^{2}}$.

The tunneling matrix element describing a process in which an electron in valley $K$ with momentum $p_{y}$ residing on sublattice $\beta$ of the nanotube hops to momentum state $\vec{k}$ and sublattice $\alpha$ in the same valley of the graphene layer is then [16]

$$
T_{\vec{k}, p_{y}}^{\alpha \beta}=\frac{t}{\sqrt{2 \pi}} \sum_{j=1}^{3} T_{j}^{\alpha \beta} \exp \left[-\frac{L^{2}}{2}\left(k_{x}-q_{j x}\right)^{2}\right] \delta_{k_{y}-p_{y}, q_{j y}},
$$

where $t \equiv t\left(k_{D}\right)$ is the Fourier transform of the tunneling amplitude $t(\vec{r})$ and $k_{D}$ is the magnitude of the Brillouin-zone corner wave vector. Here we have introduced the vectors $\vec{q}_{j}=k_{\theta}\left(\sin \left(\chi_{j}+\theta\right),-\cos \left(\chi_{j}+\theta\right)\right)$, with $k_{\theta}=2 k_{D} \sin (\theta / 2)$ and $\chi_{j}=2 \pi(j-1) / 3$, and the tunneling matrices $T_{j}$, i.e.,

$$
\begin{aligned}
& T_{1}=\left(\begin{array}{ll}
1 & 1 \\
1 & 1
\end{array}\right), \\
& T_{2}=e^{-i \overrightarrow{\mathcal{G}}_{2} \cdot \vec{d}}\left(\begin{array}{cc}
e^{-i \phi} & 1 \\
e^{i \phi} & e^{-i \phi}
\end{array}\right), \\
& T_{3}=e^{-i \overrightarrow{\mathcal{G}}_{3} \cdot \vec{d}}\left(\begin{array}{cc}
e^{i \phi} & 1 \\
e^{-i \phi} & e^{i \phi}
\end{array}\right) .
\end{aligned}
$$

Here $\phi=2 \pi / 3$ and $\overrightarrow{\mathcal{G}}_{2,3}$ are the reciprocal lattice vectors depicted in Fig. 1. For a vanishing twist angle, the tunneling Hamiltonian (2) is translationally invariant along the $y$ direction and the $y$ component of the momentum is a good quantum number. For a twist angle $\theta=\arctan (q / \sqrt{3})$, with $q$ a rational number, the $y$ component of the momentum is a good quantum number up to a reciprocal lattice vector [16]. The corresponding Hamiltonian is then periodic along the $y$ direction and can be diagonalized in terms of onedimensional Moiré bands using a plane wave expansion. ${ }^{3}$ For other twist angles, the spectrum is quasiperiodic: The corresponding wave functions are quasilocalized and tunneling is suppressed [17].

In this Rapid Communication we focus on the translationally invariant case, i.e., $\theta=0$, showing that even a rigid displacement can strongly affect nanotube electronic properties. Below we characterize how the hybridization between the nanotube and the graphene sheet alters the nanotube electronic properties by evaluating full momentum- and frequencyresolved spectral function of the nanotube [18]

$$
\mathcal{A}\left(p_{y}, \omega\right)=-\frac{1}{\pi} \sum_{\gamma} \operatorname{Im} G_{\gamma \gamma}^{R}\left(p_{y}, \omega\right),
$$

\footnotetext{
${ }^{1}$ In practice, the falloff of the tunneling strength as a function of distance from the center of the nanotube-graphene contact region will depend on nanotube shape deformation, but these details do not alter our results in an important way.

${ }^{2}$ We have tested the opposite tunneling limit, i.e., a tunneling amplitude between nanotube and graphene across the contact area between the two subsystems that falls off abruptly, and our results are not qualitatively modified (see the Supplemental Material [16]).

${ }^{3}$ We note that, in contrast to the graphene bilayer case, in our model there is only one good momentum even in the periodic limit.
} 

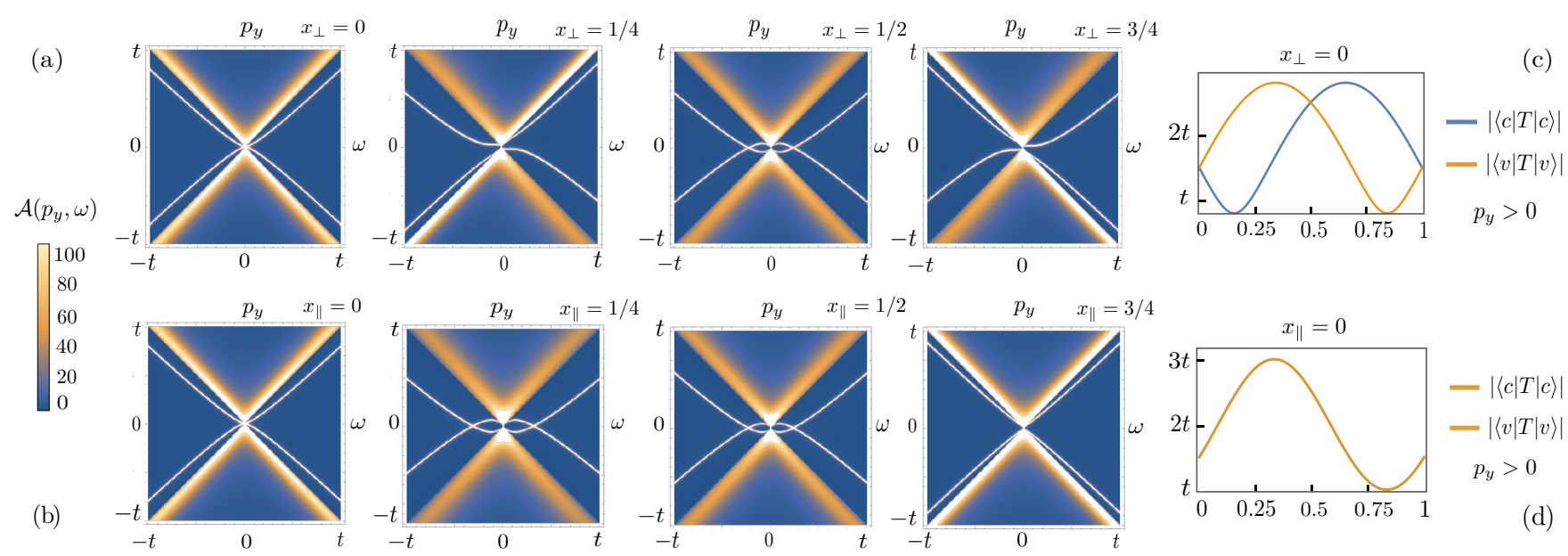

$-|\langle c|T| c\rangle|$
$-|\langle v|T| v\rangle|$
$p_{y}>0$
(d)

FIG. 2. Electronic structure of a carbon nanotube on a graphene sheet. Spectral function $\mathcal{A}\left(p_{y}, \omega\right)$ of the carbon nanotube for rigid displacements (a) along the direction transverse and (b) parallel to the nanotube axis. (c) Dependence of the (absolute value of) conduction $(|\langle c|T| c\rangle|)$ and valence $(|\langle v|T| v\rangle|)$ band hybridization matrix elements (7) at the graphene momentum $k_{x}=0$ on displacement transverse to the nanotube axis. For the dimensionless displacements $x_{\perp} \neq 0,1 / 2$, the two matrix elements have different values, explaining the asymmetry of the spectral function (a). (d) For displacements along the nanotube axis, the valence and conduction bands experience the same interaction strength, explaining the symmetry of the spectral function (b). The spectral densities, frequencies, momenta, and tunneling strength are plotted in dimensionless units, i.e., $\mathcal{A} \rightarrow(v / a) \mathcal{A}, p_{y} \rightarrow a p_{y}, \omega \rightarrow \omega(a / v)$, and $t \rightarrow t(a / v)$.

where $G_{\gamma \gamma}^{R}$ is the $\gamma \gamma$ th matrix element of the nanotube retarded Green's function, i.e.,

$$
G^{R}\left(p_{y}, \omega\right)=\lim _{\epsilon \rightarrow 0}\left[(\omega+i \epsilon) \mathcal{I}-h\left(p_{y}\right)-\Sigma\left(p_{y}, \omega\right)\right]^{-1} .
$$

Here $\mathcal{I}$ is the $2 \times 2$ identity matrix, while

$$
\Sigma_{i j}\left(p_{y}, \omega\right)=\int d k_{x}\left[T^{\dagger}\left(p_{y}\right) G_{g}^{R}\left(k_{x}, p_{y}, \eta, \omega\right) T\left(p_{y}\right)\right]_{i j}
$$

is the $i j$ th matrix element of the carbon nanotube self-energy due to hybridization with the graphene sheet. Here $G_{g}^{R}$ is the retarded Green's function of the unperturbed graphene sheet [16].

Results. In this section we present the results of a numerical evaluation of the spectral density (4) as function of a rigid displacement $\vec{d}$ of the nanotube. We distinguish translations transverse $\vec{d}_{\perp}$ and parallel $\vec{d}_{\|}$to the nanotube axis and define dimensionless translations $x_{\perp}(\|)$ by $\vec{d}_{\perp}=$ $\sqrt{3} a x_{\perp}(0,1)$ and $\vec{d}_{\|}=a x_{\|}(1,0)$, with $a$ the lattice constant of graphene. ${ }^{4}$ The electronic structure is a periodic function of $x_{\perp(\|)}$ with period 1. Results for several different values of $x_{\|(\perp)}$ at $t=100 \mathrm{meV},{ }^{5} L=10 \pi \mathrm{nm}$, and $\eta=0$ are summarized in Figs. 2(a) and 2(b). Figure 2(a) shows the dependence of the spectral density (4) on translations transverse to the nanotube axis. The isolated bands illustrate the strong influence of hybridization on the nanotube band

\footnotetext{
${ }^{4}$ In the absence of twist, the unit cell is defined by the honeycomb lattice of graphene.

${ }^{5}$ Our results do not qualitatively depend on the tunneling strength $t$, as long as the latter is weaker than the energy separation between nanotube minibands ( $\sim 1 \mathrm{eV}$ for our parameters). A higher (weaker) tunneling strength would result in an increased (decreased) intensity of the spectral density.
}

dispersion, while the continuous spectra reflect leakage of the graphene sheet orbitals onto the nanotube. We observe that the nanotube Dirac velocities are generically reduced by level repulsion with graphene sheet orbitals and that the linear dispersion of isolated nanotubes can be strongly distorted. For a general translation $x_{\perp} \in(0,1) \neq 0,1 / 2$, the nanotube spectrum displays an asymmetry between right goers and left movers (i.e., corresponding to valley-projected time-reversal symmetry breaking) and the graphene spectral weight shows a corresponding asymmetry. On the other hand, as illustrated in Fig. 2(b), symmetric spectra and spectral weight are maintained for any translation along the nanotube axis.

Most features of Figs. 2(a) and 2(b) can be understood by examining the hybridization matrix elements between the nanotube and graphene conduction and valence bands, while setting the $x$ component of the graphene momentum $k_{x} \rightarrow 0$. This suffices to capture the largest contribution to the spectral density, which is due to energy-conserving processes in which electrons tunnel from the nanotube to the graphene layer and vice versa. Since the hopping term (2) preserves the $y$ component of momentum, energy is conserved during tunneling only when $k_{x}=0$. The hybridization matrix elements between graphene and nanotube conduction and valence bands at $k_{x}=0$ are

$$
\begin{aligned}
& T_{c c} \equiv\langle c|T| c\rangle=\frac{1}{2}\left[T^{A A}+T^{B B}+i\left(T^{A B}-T^{B A}\right)\right], \\
& T_{v v} \equiv\langle v|T| v\rangle=\frac{1}{2}\left[T^{A A}+T^{B B}+i\left(T^{B A}-T^{A B}\right)\right]
\end{aligned}
$$

for $p_{y}>0$, while for $p_{y}<0 \quad T_{c c} \leftrightarrow T_{v v}$. Here we have dropped the momentum subscripts for the sake of brevity, i.e., $T^{\alpha \beta}=T_{\vec{k}, p_{y}}^{\alpha \beta}$ [Eq. (2)]. Figures 2(c) and 2(d) illustrate the variation of the matrix elements in Eq. (7) upon transverse and parallel displacement, respectively. Considering, for instance, $x_{\perp}=3 / 4$ and $p_{y}>0$, one finds that the repulsive interaction between conduction bands is much larger than the one 

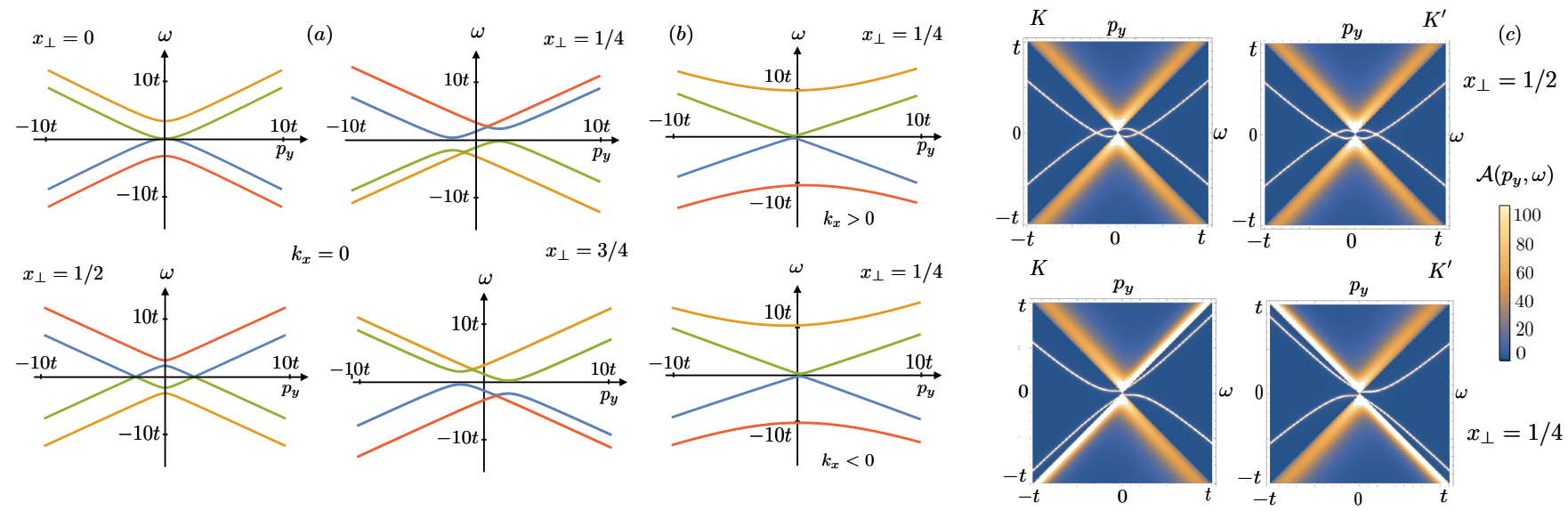

FIG. 3. Four-band model and valley-dependent spectral functions. (a) Energy spectrum of the $4 \times 4$ Hamiltonian (8) for graphene momentum $k_{x}=0$ and different values of dimensionless displacement $x_{\perp}$. Far from the Dirac point, the spectrum displays the same features observed in the spectral function [see Fig. 2(a)]. However, in contrast with the spectral function, the four-band spectrum exhibits an energy gap. (b) Energy spectrum of the $4 \times 4$ Hamiltonian (8) for a finite $k_{x}$ and $x_{\perp}=1 / 4$. For $k_{x}>0(<0)$, the gap closes at the left (right) of the Dirac point. The total contribution of electronic states with finite $k_{x}$ gives rise to a gap closing at the Dirac point, which is displayed by the spectral density in Fig. 2(a). (c) Nanotube spectral density $\mathcal{A}\left(p_{y}, \omega\right)$ at the valleys $K$ and $K^{\prime}$, which are related by time-reversal symmetry. A valley-projected time-reversal symmetric spectrum is degenerate for $K$ and $K^{\prime}$ (top panels). When valley-projected time-reversal symmetry is broken (bottom panels), a right-going state at momentum $p_{y}$ in one valley still has a left-going degenerate Kramers partner at momentum $-p_{y}$ in the other valley. The spectral densities, frequencies, momenta, and tunneling strength are plotted in dimensionless units, i.e., $\mathcal{A} \rightarrow(v / a) \mathcal{A}$, $p_{y} \rightarrow a p_{y}, \omega \rightarrow \omega(a / v)$, and $t \rightarrow t(a / v)$.

between valence bands, i.e., $\left|T_{c c}\right| \gg\left|T_{v v}\right|$. As a result, the velocity renormalization of the nanotube conduction band is larger than for the valence band. Larger velocity reductions also lead to smaller amplitudes for the graphene sheet orbitals on the nanotube.

Although the strongest trends in Fig. 2(a) can be understood by examining level repulsion separately for conduction and valence bands, the behavior near $p_{y}=0$ involves all four bands. The full four-band Hamiltonian of the coupled bilayers is

$$
\mathcal{H}=\left(\begin{array}{cccc}
0 & h_{g 12} & T_{11} & T_{12} \\
h_{g 21} & 0 & T_{21} & T_{22} \\
T_{11}^{*} & T_{21}^{*} & 0 & h_{12} \\
T_{12}^{*} & T_{22}^{*} & h_{21} & 0
\end{array}\right) .
$$

Figure 3(a) shows that diagonalizing Eq. (8) at $k_{x}=0$ explains most of the behavior seen Fig. 2(a), although some gaps in the four-band spectrum do not survive in the nanotube spectral function. The absence of such gap can be explained by accounting for graphene states with $k_{x} \neq 0$. Figure 3(b) shows that diagonalizing Eq. (8) for a finite $k_{x}$ leads to a gap closing to the left and right of the Dirac point for $k_{x}>0$ and $k_{x}<0$, respectively. Adding these two contributions results in a gap closing at the Dirac point, i.e., $p_{y}=0$. Finite momentum contributions lead also to a shift of the graphenelike conduction and valence bands to higher energies (in absolute magnitude). This is reflected by the spectral weight broadening we observe in correspondence to the graphene Dirac cone.

Another interesting feature of our results is the broken valley degeneracy of the spectrum, which we observe for any transverse translation (with the exception of $x_{\perp}=0,1 / 2$ ), but never when considering displacements parallel to the nanotube axis. This property of the spectrum follows from the symmetries of our model. Time-reversal symmetry constrains the spectrum at momentum $p_{y}$ in the $K$ valley to equal the spectrum in the $K^{\prime}$ valley at momentum $-p_{y}$, as can be seen in Fig. 3(c). The valley-projected spectrum is particle-hole symmetric for any displacement. When the valley-projected spectrum is time-reversal symmetric, the two valleys are degenerate at each $p_{y}$. When valley-projected time-reversal symmetry is absent, on the other hand, the spectrum of the $K$ valley can be mapped into the $K^{\prime}$ valley by setting $p_{y} \rightarrow-p_{y}$.

Discussion and outlook. In this work we have established a low-energy effective model for a carbon nanotube on top of a graphene layer, which is valid for any displacement or rotation of the nanotube axis. Depending on tube orientation and chirality, the heterojunction Hamiltonian can be translationally invariant, periodic, or quasiperiodic. In the translationally invariant case, we show that, even at a vanishing twist angle, rigid displacements of the nanotube with respect to the graphene layer can strongly alter the electronic properties of the former. For instance, a rigid displacement of the nanotube can break particle-hole symmetry, ${ }^{6}$ lift the degeneracy between the two valleys, and strongly alter the carrier velocity.

These features, which can be explained through a fourband model and symmetry considerations, might be probed experimentally by nanospot angle-resolved photoemission spectroscopy, Raman spectroscopy, or momentum- and

\footnotetext{
${ }^{6}$ Note that such particle-hole symmetry breaking does not lead to any net charge transfer since the particle-hole symmetry of the valley-projected spectrum is preserved (see the Supplemental Material [16]).
} 
energy-resolved tunneling spectroscopy [19]. Controlled growth or deposition of nanotubes on a substrate has been already demonstrated [20,21] and nanotubes can be laterally displaced on top of a graphene layer by, e.g., atomic force microscopy [22], allowing our predictions to be tested experimentally.

Our work suggests that atomic force manipulation of carbon nanotubes on graphene substrates can radically alter electronic properties, leading in some cases to strong correlations related to flattened nanotube bands, and to interesting modified electronic structures with radically different quasiparticle velocities in different valleys.
Finally, the nanotube might experience radial or axial deformation when lying on a substrate [23-27]. Following Ref. [28], we have investigated the effects of strain relaxation and concluded that our results are not qualitatively altered by it [16]. While our model relies on Ref. [12], more accurate descriptions of the interlayer tunneling should be investigated in future work [29].

Acknowledgments. The authors thank Y. Yuval for insightful discussions. B.F. was supported by the Dutch Science Foundation through a Rubicon grant. A.H.M. was supported by DOE Grant No. DE-FG02-02ER45958 and Welch Foundation Grant No. TBF1473.
[1] A. H. Castro Neto, F. Guinea, N. M. R. Peres, K. S. Novoselov, and A. K. Geim, Rev. Mod. Phys. 81, 109 (2009).

[2] T. Ando, J. Phys. Soc. Jpn. 74, 777 (2005).

[3] X. Huang, Z. Yin, S. Wu, X. Qi, Q. He, Q. Zhang, Q. Yan, F. Boey, and H. Zhang, Small 7, 1876 (2011).

[4] P. Avouris, M. Freitag, and V. Perebeinos, Nat. Photon. 2, 341 (2008).

[5] P. L. McEuen, M. S. Fuhrer, and H. Park, IEEE Trans. Nanotechnol. 1, 78 (2002).

[6] J. J. Baumberg, Nat. Mat. 16, 877 (2017).

[7] K. S. Novoselov, A. K. Geim, S. V. Morozov, D. Jiang, M. I. Katsnelson, I. V. Grigorieva, S. V. Dubonos, and A. A. Firsov, Nature (London) 438, 197 (2005); Y. Zhang, Y.-W. Tan, H. L. Stormer, and P. Kim, ibid. 438, 201 (2005).

[8] K. S. Novoselov, A. K. Geim, S. V. Morozov, D. Jiang, Y. Zhang, S. V. Dubonos, I. V. Grigorieva, and A. A. Firsov, Science 306, 666 (2004).

[9] E. McCann and M. Koshino, Rep. Prog. Phys. 76, 056503 (2013).

[10] Y. Cao, V. Fatemi, S. Fang, K. Watanabe, T. Taniguchi, E. Kaxiras, and P. Jarillo-Herrero, Nature (London) 556, 43 (2018).

[11] Y. Cao, V. Fatemi, A. Demir, S. Fang, S. L. Tomarken, J. Y. Luo, J. D. Sanchez-Yamagishi, K. Watanabe, T. Taniguchi, E. Kaxiras, R. C. Ashoori, and P. Jarillo-Herrero, Nature (London) 556, 80 (2018); M. Yankowitz, S. Chen, H. Polshyn, Y. Zhang, K. Watanabe, T. Taniguchi, D. Graf, A. F. Young, and C. R. Dean, Science 363, 1059 (2019).

[12] R. Bistritzer and A. H. MacDonald, Proc. Natl. Acad. Sci. USA 108, 12233 (2011).

[13] N. Kainaris and S. T. Carr, Phys. Rev. B 92, 035139 (2015).

[14] G. E. Volovik, J. Supercond. Nov. Magn. 26, 2887 (2013).
[15] R. Saito, G. Dresselhaus, and M. S. Dresselhaus, Physical Properties of Carbon Nanotubes (Imperial College Press, London, 1998).

[16] See Supplemental Material at http://link.aps.org/supplemental/ 10.1103/PhysRevResearch.2.022041 for a detailed discussion.

[17] A. H. MacDonald, in Interfaces, Quantum Wells and Superlattices, edited by R. Leavers and R. Taylor (Plenum, New York, 1988); J. Bellisard, B. Iochum, E. Scoppola, and D. Testard, Commun. Math. Phys. 125, 527 (1989).

[18] H. Bruus and K. Flesberg, Many-Body Quantum Theory in Condensed Matter Physics (Oxford University Press, Oxford, 2004).

[19] J. Jang, H. M. Yoo, L. N. Pfeiffer, K. W. West, K. W. Baldwin, and R. C. Ashoori, Science 358, 901 (2017).

[20] J. Xiao, S. Dunham, P. Liu, Y. Zhang, C. Kocabas, L. Moh, Y. Huang, K.-C. Hwang, C. Lu, W. Huang, and J. A. Rogers, Nano Lett. 9, 4311 (2009).

[21] J. Gramich, A. Baumgartner, M. Muoth, C. Hierold, and C. Schönenberger, Phys. Status Solidi B 252, 2496 (2015).

[22] M. Seydou, Y. J. Dappe, S. Marsaudon, J.-P. Aimé, X. Bouju, and A.-M. Bonnot, Phys. Rev. B 83, 045410 (2011).

[23] B. Yakobson and P. Avouris, Top. Appl. Phys. 80, 287 (2001).

[24] L. Yang and J. Han, Phys. Rev. Lett. 85, 154 (2000).

[25] T. Hertel, R. E. Walkup, and P. Avouris, Phys. Rev. B 58, 13870 (1998).

[26] J. Cao, Q. Wang, and H. Dai, Phys. Rev. Lett. 90, 157601 (2003).

[27] E. D. Minot, Y. Yaish, V. Sazonova, J.-Y. Park, M. Brink, and P. L. McEuen, Phys. Rev. Lett. 90, 156401 (2003).

[28] S. Carr, S. Fang, Z. Zhu, and E. Kaxiras, Phys. Rev. Res. 1, 013001 (2019).

[29] X. Lin and D. Tománek, Phys. Rev. B 98, 081410(R) (2018); S. Fang and E. Kaxiras, ibid. 93, 235153 (2016); V. Perebeinos, J. Tersoff, and P. Avouris, Phys. Rev. Lett. 109, 236604 (2012). 